\title{
Descolamento viteliforme macular associado a drusas da lâmina basal: relato de caso
}

\author{
Vitelliform maculardetachmentassociated with basal laminardrusen: \\ case report
}

\author{
Maria Teresa Brizzi Chizzotti Bonanomi ${ }^{1}$ \\ Otacílio de Oliveira Maia Júnior ${ }^{2}$ \\ Leda Mine Takei Lower ${ }^{3}$ \\ Yoshitaka Nakashima ${ }^{4}$
}

\section{RESUMO}

Lesão viteliforme da mácula é mais observada nas distrofias de Best e foveomacular do adulto. No presente estudo, os autores relatam caso raro de descolamento viteliforme macular associado a drusas da lâmina basal, acompanhado no Hospital das Clínicas da Faculdade de Medicina da Universidade de São Paulo há aproximadamente quatro anos, avaliado periodicamente com exames complementares. Os autores descrevem aspectos relevantes desta doença como etiopatogenia, evolução, diagnósticos diferenciais e tratamento.

Descritores: Membrana basal; Angiofluoresceinografia; Fundo de olho; Macula lutea/ lesões; Epitélio pigmentado ocular/patologia; Drusa retiniana/complicações; Fóvea central; Descolamento retiniano/etiologia; Relatos de casos [tipo de publicação]

\section{INTRODUÇÃO}

Drusas da lâmina basal foram primeiramente descritas por Gass, em 1985, que as definiu como espessamentos difusos da membrana basal adjacentes ao epitélio pigmentado da retina $(\mathrm{EPR})^{(1)}$. Estas drusas acometem adultos jovens bilateralmente, apresentando-se como inúmeras lesões nodulares sub-retinianas pequenas $(25 \mu \mathrm{m}$ a $75 \mu \mathrm{m})$, podendo estar agrupadas na mácula, de aspecto amarelado e uniforme. Nestas drusas, não há acúmulo de material entre a membrana de Bruch e a membrana basal do EPR como ocorre nas drusas clássicas, mas espessamento difuso e nodular da membrana basal do EPR, sendo bem mais visíveis na angiofluoresceinografia (AFG) que na biomicroscopia ${ }^{(1)}$. É descrito aspecto de "estrelas no céu desaparecendo", observado pela fluorescência no início da fase arteriovenosa, sendo que este aspecto desaparece no decorrer desta fase e em seguida surgem drusas clássicas que fluorescem no final ${ }^{(2)}$.

O descolamento viteliforme macular, decorrente das drusas da lâmina basal, ocorre mais em mulheres com idade avançada. Este descolamento pode mimetizar as lesões observadas na distrofia de Best e na distrofia foveomacular do adulto, porém o diagnóstico diferencial é estabelecido pelo contexto clínico e alterações em exames complementares.

No presente estudo, os autores relatam um caso de descolamento viteliforme da mácula, que foi encaminhado com diagnóstico prévio de distrofia de Best e, posteriormente, firmado que a causa base do descolamento foi secundária às drusas da lâmina basal, demonstrando a importância do conhecimento desta entidade nosológica com seus diagnósticos diferenciais, tratamento e prognóstico. 


\section{RELATO DE CASO}

ACS, 65 anos, masculino, advogado aposentado, natural e procedente de São Paulo.

Reprovado no exame médico para carteira de motorista, procurou oftalmologista que detectou lesão no fundo do olho fazendo diagnóstico de distrofia de Best.

Encaminhado para avaliação em nosso serviço em 05/07/2000, apresentava acuidade visual com melhor correção no olho direito $(O D)=20 / 80$ e no olho esquerdo $(O E)=20 / 60$. Antecedentes individuais e familiares irrelevantes. Biomicroscopia normal em ambos os olhos (AO), exceto por lesão verrucosa na margem da pálpebra superior do OD. A pressão intra-ocular era de $16 \mathrm{mmHg}$ no OD e de $14 \mathrm{mmHg}$ no OE.

O fundo de olho (FO) apresentava lesão amarelada circular de aproximadamente dois diâmetros papilares na mácula em $\mathrm{AO}$, com leve assimetria, mais alterado no OD que apresentava pequenas lesões satélites (Figura 1).

O eletrooculograma (EOG) mostrou resposta de 184,21\% para o OD e de $186,67 \%$ para o OE, ambos dentro do limite inferior da normalidade. O eletroretinograma (ERG) mostrou em AO; no exame escotópico: diminuição da amplitude da onda b ao estímulo azul sem alterações nos outros estímulos; no exame fotópico e no "Flicker": respostas dentro dos limites da normalidade.

$\mathrm{O}$ paciente recusou-se inicialmente à realização da AFG.

Após quatro meses, queixava-se de piora da visão em AO, apresentando acuidade visual de 20/200 em AO com metamorfopsia no OE. Ao exame de FO, apenas a mácula deste olho havia alterado, com reabsorção do depósito amarelado na metade inferior da lesão (Figura 2A).

Submetido à AFG que mostrou tempos circulatórios nor- mais, com hiperfluorescência brilhante de múltiplos pontos, no início da fase arteriovenosa, em todo o pólo posterior de AO. No decorrer do exame, o descolamento inicialmente hipofluorescente, devido ao bloqueio da fluorescência da coróide pela exsudação, tornou-se progressivamente hiperfluorescente. Este aumento ocorreu a partir das bordas do descolamento até que toda área descolada ficasse com hiperfluorescência brilhante especialmente no OD (Figura 3). No OE, foi possível individualizar drusas clássicas na parte inferior do descolamento, onde não havia mais o material amarelo no espaço subretiniano.

Com este quadro clínico, foi firmado o diagnóstico de descolamento viteliforme da mácula associado a drusas de lâmina basal em AO. O paciente foi mantido sob observação periódica.

$\mathrm{Na}$ evolução do quadro, a reabsorção do material subretiniano no OE foi mais intensa e, depois de aproximadamente quatro anos sem instituir qualquer modalidade terapêutica, a acuidade visual era de 20/200 no OD e de 20/80 no OE, associada à atrofia geográfica em AO (Figura 2B).

\section{DISCUSSÃO}

O descolamento viteliforme exsudativo da mácula é uma entidade incomum que ocorre em pacientes portadores de drusas da lâmina basal do $\operatorname{EPR}^{(1)}$. Nestas drusas, não há acúmulo de material entre a membrana de Bruch e a membrana basal do EPR, como ocorre nas drusas clássicas, mas sim espessamento difuso e nodular da membrana basal do EPR. Estas drusas são bem mais visíveis na AFG que na biomicroscopia. O angiograma previamente descrito deste paciente corresponde à descrição da literatura ${ }^{(1-2)}$.

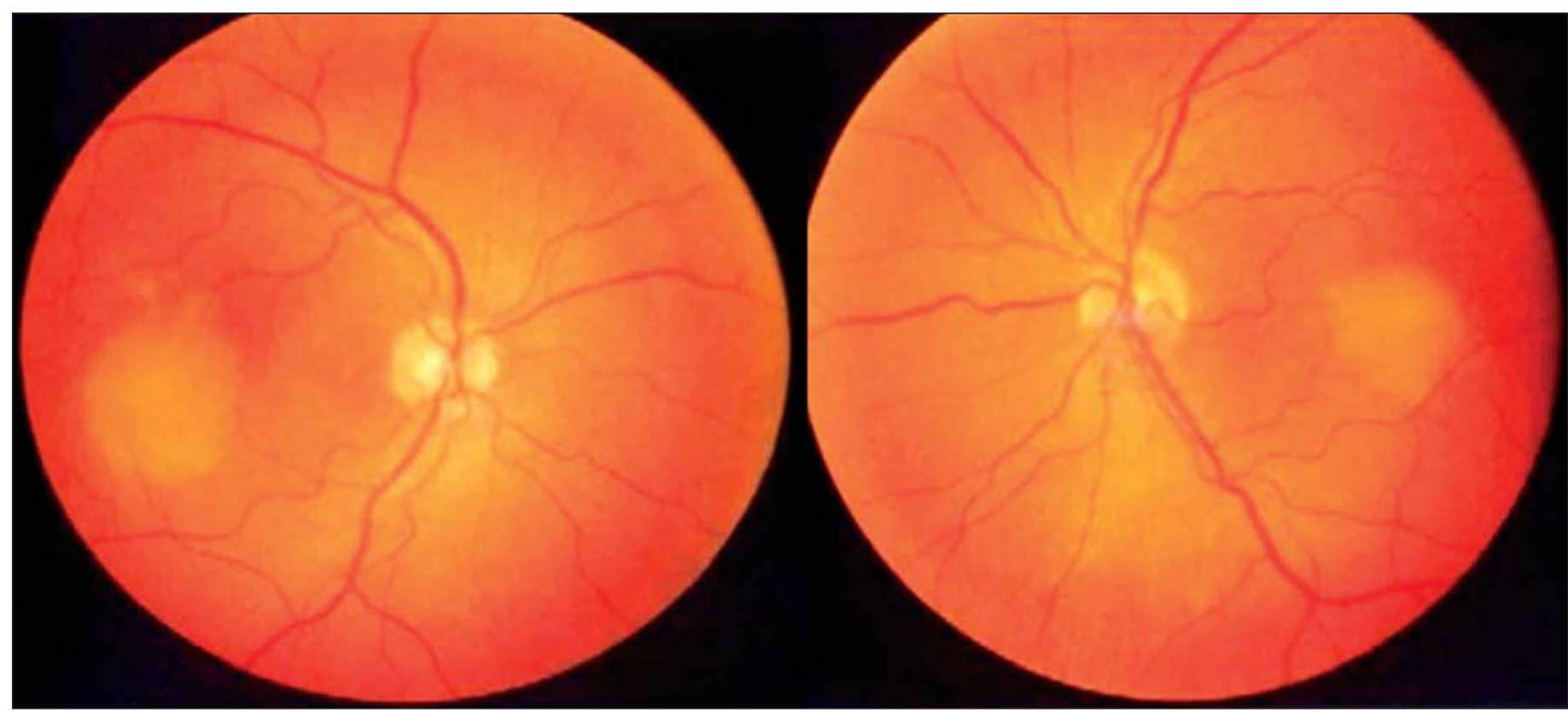

Figura 1 - Descolamento viteliforme macular em AO. [Nota: Desconsiderar a presença de artefato (reflexo do retinógrafo) no centro das duas fotos] 

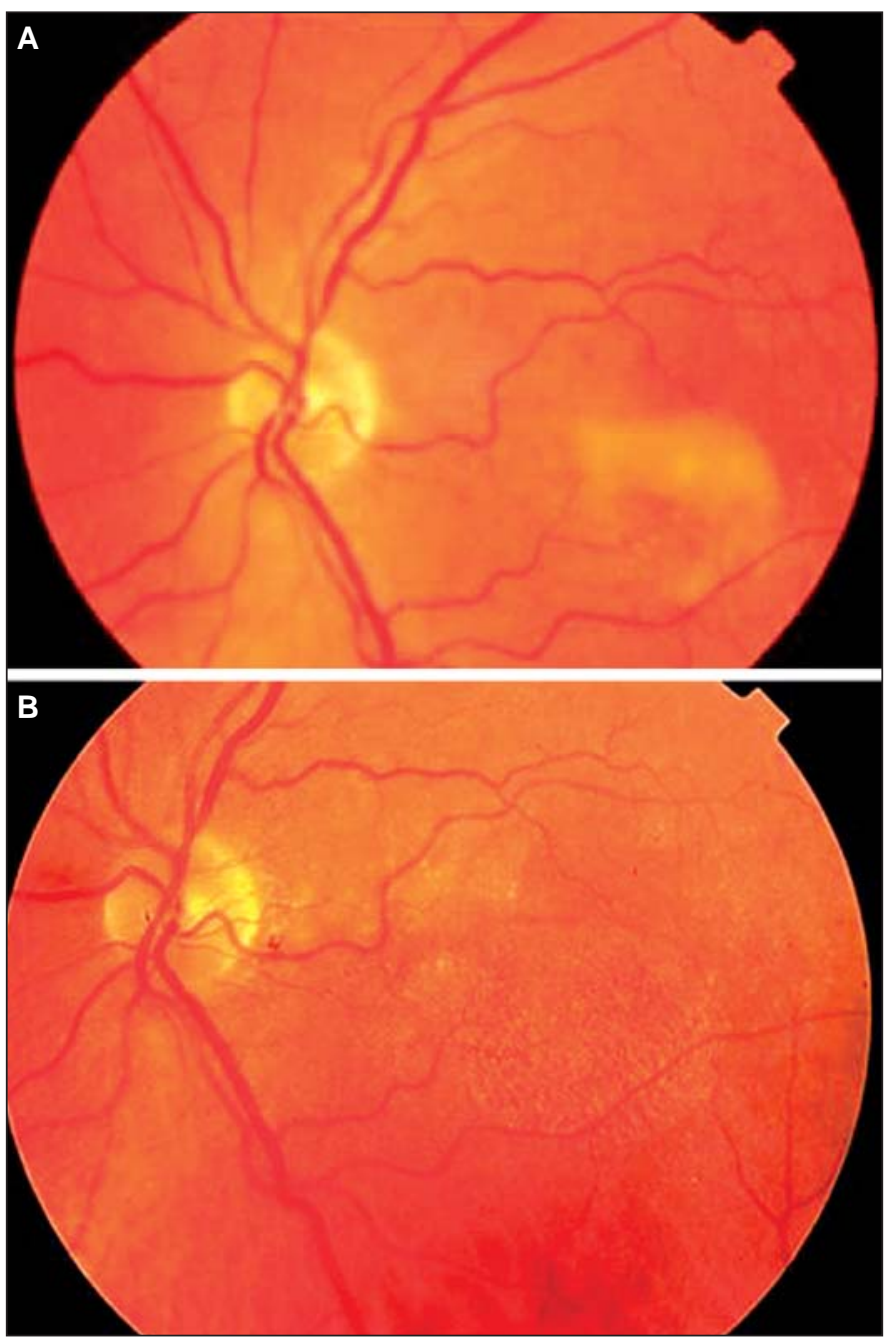

Figura 2 - Evolução do OE. A: Reabsorção parcial do líquido subretiniano, apenas na parte inferior da lesão; B: Aspecto no final do período de seguimento com a zona de atrofia geográfica correspondente à antiga área de descolamento viteliforme macular

A associação com drusas exsudativas está descrita em pacientes acima de $50 \operatorname{anos}^{(1-2)}$. É característica também a associação com descolamentos exsudativos de retina com a cor variando do branco opalescente ao amarelo vivo, ao contrário do que ocorre na degeneração macular relacionada à idade, que apresenta descolamento do EPR. Quando bem delimitados e densamente amarelados, como no presente caso, estes descolamentos exsudativos podem mimetizar distrofia de Best e distrofia foveomacular do adulto. Na distrofia de Best, a história familiar com herança autossômica dominante é positiva e o exame eletrodiagnóstico é peculiar: EOG anormal e ERG normal $^{(3)}$. Na distrofia foveomacular do adulto, é característica uma lesão amarelada na mácula, arredondada ou ovalada, bilateral, atingindo cerca de 1/3 de diâmetro papilar e, em geral, associada a um ponto pigmentado central ${ }^{(4-5)}$. Este quadro inicia-se entre a $4^{\underline{a}}$ e $6^{\underline{a}}$ décadas de vida com perda visual leve e a AFG é característica, evidenciando hipofluorescência central com anel hiperfluorescente nas bordas da lesão, des-


Figura 3 - AFG do OD com a hiperfluorescência puntiforme das drusas da lâmina basal do epitélio pigmentado da retina. A: Fase arteriovenosa inicial com hipofluorescência de toda a área do descolamento viteliforme e suas lesões satélites; B: Com o passar do tempo, as lesões satélites visíveis mantêm a hipofluorescência e há hiperfluorescência tardia por acúmulo de corante na cavidade do descolamento viteliforme

crito como sinal do eclipse ${ }^{(6)}$. O EOG pode ser normal ou alterado e existem, na literatura, relatos de casos de ocorrência familiar da doença ${ }^{(7)}$. As alterações histopatológicas descritas previamente mostram perda focal de fotorreceptores, atrofia do EPR e quantidade aumentada de lipofuscina ${ }^{(5,8-9)}$.

Nos casos de descolamento viteliforme associados com drusas da lâmina basal, a acuidade visual pode melhorar com reabsorção do líquido sub-retiniano, sendo que tratamento com laser das zonas periféricas ao descolamento, onde parece haver o início de "pooling", provavelmente não interfere na evolução do quadro $^{(1)}$. Neste paciente, o OD apresentava 20/200 sem sinais de membrana neovascular da coróide ou atrofia do EPR, sendo possível uma melhora espontânea da 
lesão. A acuidade visual do olho esquerdo foi de 20/200, com distorção na tela de Amsler devido a uma provável absorção assimétrica do líquido, associada à atrofia geográfica. Além da diminuição da acuidade visual e da metamorfopsia, são descritas discromatopsias no eixo azul-amarelo, alterações na sensibilidade de contraste e no limiar de sensibilidade foveal ${ }^{(2)}$. O prognóstico visual para estes pacientes é bom, se não houver complicação por membrana neovascular da coróide (MNC), o que pode ocorrer em $31 \%$ dos $\operatorname{casos}^{(2)}$.

Em suma, drusas da lâmina basal predispõem ao descolamento viteliforme macular, sendo importante o reconhecimento e seguimento dos pacientes acometidos por estas lesões. Para firmar o diagnóstico, é necessário excluir doenças que cursam com lesões viteliformes, como distrofias de Best e foveomacular do adulto, através da história clínica e exames complementares. Depois de instalado o quadro de descolamento viteliforme, secundário às drusas da lâmina basal, o tratamento é desnecessário, pois a resolução, na maioria das vezes, é espontânea. Entretanto, pode-se desenvolver MNC, atrofia do EPR ou buraco de mácula, causando baixa da acuidade visual.

\section{ABSTRACT}

The vitelliform macular lesion is usually observed in Best's disease and the adult type of foveomacular dystrophy. We describe a rare case of macular vitelliform lesion in association with basal laminar drusen. The patient has been followed at the Hospital das Clínicas da Faculdade de Medicina da Universidade de São Paulo for the last four years. The differential diagnosis, clinical evolution, pathogenesis and treatment of this rare condition are discussed.

Keywords: Basement membrane; Fluorescein angiography; Fundus oculi; Macula lutea/injuries; Pigment epithelium of eye/ pathology; Retinal drusen/complications; Fovea centralis; Retinal detachment/etiology; Case reports [publication type]

\section{REFERÊNCIAS}

1. Gass JD, Jallow S, Davis B. Adult vitelliform macular detachment occurring in patients with basal laminar drusen. Am J Ophthalmol. 1985;99(4):445-59.

2. Cohen SY, Meunier I, Soubrane G, Glacet-Bernard A, Coscas GJ. Visual function and course of basal laminar drusen combined with vitelliform macular detachment. Br J Ophthalmol. 1994;78(6):437-40.

3. Cordeiro F, Bonomo PP. Distrofias viteliformes da mácula. In: Bonomo PP, Cunha SL. Doenças da mácula. São Paulo: Roca; 1993. p.63-8.

4. Bloom LH, Swanson DE, Bird AC. Adult vitelliform macular degeneration. $\mathrm{Br}$ J Ophthalmol. 1989;65(11):800-1.

5. Patrinely JR, Lewis RA, Font RL. Foveomacular vitelliform dystrophy, adult type. A clinicopathologic study including electron microscopic observations. Ophthalmology. 1985;92(12):1712-8.

6. Vianna RNG. Lesão macular viteliforme. Rev Bras Oftalmol. 1996;55(11):815-21.

7. Hittner HM, Ferrel RE, Borba RP, Justice JJ Jr. Atypical vitelliform macular distrophy in a 5-generation family. Br J Ophthalmol. 1984;68(3):199-207.

8. Gass JD. A clinicopathologic study of a peculiar foveomacular dystrophy. Trans Am Ophthalmol Soc. 1974;72:139-56.

9. Araújo PCM, Cerqueira VB, Saud E, Pereira MB, Moraes Júnior HV. Lesão macular viteliforme - relato de caso. Rev Bras Oftalmol. 2002;61(11):820-4.

\section{Cursa de Lentes de Contato e Ortaceratologia da Sante Case do Rio de Jeneiro}

\section{7 a 19 de Rgosto de 2006 Río de Janeiro - RJ}

INFORMAÇŨES

Tel.: (2l) 2554-1018 com Cida • E-mail: tboldrim@hotmail.com 